STRUCTURAL SCIENCE CRYSTAL ENGINEERING MATERIALS

ISSN 2052-5206

Received 21 May 2021

Accepted 21 May 2021

Keywords: organic molecular crystal; thermal expansion; X-ray diffraction; crystal engineering.
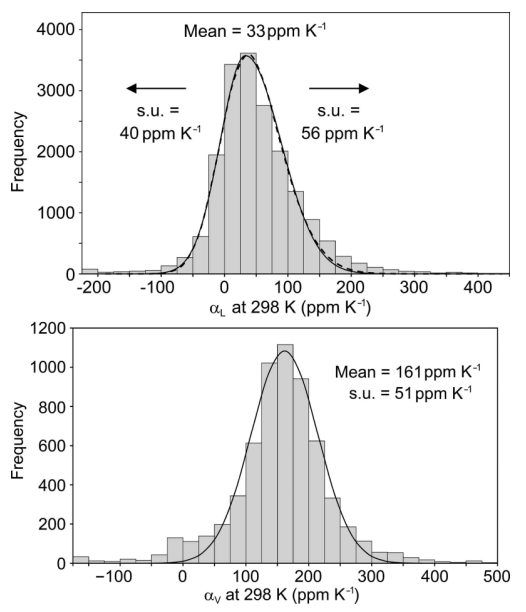

X-ray diffraction, one of the best techniques to study thermal expansion of crystalline materials, has made it easy to rationalize various properties exhibited by different compounds. Advanced X-ray diffraction techniques significantly contribute to the endeavor of crystal engineers to design a new material with desired properties. Indeed, a crystallographic database is the foremost important tool for a crystal engineer to understand intermolecular interactions between the molecules in crystals. The Cambridge Structural Database (CSD) is presently the depository of more than one million crystal structures of organic and metal-organic compounds mostly determined by single-crystal X-ray diffraction. The CSD is routinely used for different purposes by the crystal engineering community and materials scientists worldwide. This scientific commentary provides a brief insight into thermal expansion of organic molecular crystals with reference to the recent article by Andrew Bond (2021) published in Acta Crystallographica Section B on a CSD survey of thermal expansion in organic molecular crystals.

Expansion of material upon heating is a general phenomenon which is commonly known as Positive Thermal Expansion (PTE). This can be simply explained by anharmonic vibration of a diatomic bond with increasing temperature (Evans, 1999). However, an unusual phenomenon occurs when a material contracts upon heating - known as Negative Thermal Expansion (NTE). Unusual thermal expansion may also occur in cases of neither expansion nor contraction of a material which is known as Zero Thermal Expansion (ZTE). While the study of thermal expansion is extremely important to understand the structural safety and accuracy in many engineering fields such as aerospace, civil, electronic engineering and precision instruments, design of materials with desired thermal expansion is highly challenging because the underlying mechanism for NTE varies from material to material. A database study may help in understanding thermal expansion behavior of materials in general. In this context, the article by Bond (2021) addresses the general distribution of coefficients of thermal expansion (CTE) of specifically organic crystalline compounds by systematic analysis of variable-temperature single-crystal X-ray diffraction data available in the CSD. Although the thermal expansion of a large number of inorganic and hybrid materials is known, this study by Bond (2021) focuses on organic crystalline materials for which crystal structures are determined.

In order to extract the crystal structures of organic compounds deposited in the CSD, determined at a minimum of two different temperatures, the author has used a Python API and some constraints to focus only on organic molecular crystals. Some of the crystal structures in the extracted data set are inconsistent with the calculation of thermal expansion. This inconsistency arises probably due to the wrong temperature of data collection being reported. It may also happen that the reported structures were determined using different crystals at different temperatures. To avoid this inconsistency it is, therefore, necessary to use the same crystal in the same instrument for data collection by variable-temperature single-crystal X-ray diffraction to study the thermal expansion of crystalline materials.

Bond has used the PASCal program (Cliffe \& Goodwin, 2012) to calculate CTE (both volumetric and axial) of non-orthogonal crystal systems. This survey revealed both volumetric and axial PTE of organic compounds in general. There are few exceptions which show unusual thermal expansion including NTE and large PTE (Das et al., 2010). Applying linear fit methodology, this study shows a mean volumetric expansion coeffi- 


\section{scientific commentaries}

cient of 161 p.p.m. $\mathrm{K}^{-1}$ with a standard deviation of 51 p.p.m. $\mathrm{K}^{-1}$, whereas principal expansion coefficients are described by two half normal distributions centered on 33 p.p.m. $\mathrm{K}^{-1}$ with standard deviations of 40 p.p.m. $\mathrm{K}^{-1}$ (lower side) and 56 p.p.m. $\mathrm{K}^{-1}$ (upper side).

Although NTE is known for many inorganic materials, isotropic NTE and ZTE have not been observed in any organic material to date. While uniaxial NTE has been observed in a few organic materials and biaxial NTE is rare in organic compounds, volumetric expansion of organic compounds is always positive. The study of thermal expansion properties of organic materials has received much attention in recent years emphasizing the mechanism of unusual thermal expansion (Das et. al., 2010; Alimi et al., 2018; Liu et al., 2019). While certain packing arrangement induces anomalous thermal expansion in some organic compounds (Das et. al., 2010), in some organic compounds transverse vibration of atoms leads to the unusual thermal expansion (Negi et al.,
2021). Nevertheless, the goal to design an organic molecule with controlled thermal expansion is far from reality. The present database (CSD) does not contain sufficient information and more data related to thermal expansion of organic compounds is required to understand the unusual phenomenon.

\section{References}

Alimi, L. O., Lama, P., Smith, V. J. \& Barbour, L. J. (2018). CrystEngComm, 20, 631-635.

Bond, A. D. (2021). Acta Cryst. B77, 357-364.

Cliffe, M. J. \& Goodwin, A. L. (2012). J. Appl. Cryst. 45, 1321-1329.

Das, D., Jacobs, T. \& Barbour, L. J. (2010). Nat. Mater. 9, 36-39.

Evans, J. S. O. (1999). J. Chem. Soc. Dalton Trans. pp. 3317-3326.

Liu, H., Gutmann, M. J., Stokes, H. T., Campbell, B. J., Evans, I. R. \& Evans, J. S. O. (2019). Chem. Mater. 31, 4514-4523.

Negi, L., Kumar, S., Dwivedi, B. \& Das, D. (2021). Cryst. Growth Des. 21, 1428-1433. 